Approved for public release, distribution unlimited.

\title{
EXPOSURE OF PRIMATES FOR ONE Y YEAR TO \\ ELECTRIC AND MAGNETIC FIELDS ASSOCIATED WITH \\ ELF COMMUNICATIONS SYSTEMS
}

James D. Grissett, James L. Kupper, Matthew J. Kessler,

Richard J. Brown, George D. Prettyman, Larry L. Cook, and Toby A. Griner

Naval Medical Research and Development Command XSB09.ED6.6-B1

Approved by

Ashton Graybiel, M.D.

Assistant for Scientific Programs
Released by

Captain R.E. Mitchel, MC USN Commanding Officer

7 November 1977

NAVAL AEROSPACE MEDICAL RESEARCH LABORATORY

PENSACOLA, FLORIDA 32508 